\title{
A Dynamic, Mathematical Model for Quantitative \\ Glycoprofiling using Label-free Lectin Microarrays
}

Daniel P. Salem, Justin T. Nelson, Sojin Kim and Michael S. Strano*

Department of Chemical Engineering, Massachusetts Institute of Technology, Cambridge, MA

02139

*Corresponding author. Email: strano@mit.edu, Phone: (617) 324-4323

\section{Supporting Information}

The output of the lectin microarray and the proposed kinetic model enable the determination of $\mathbf{G}$, or the vector of all glycan concentrations. Given a single protein mixture, the determination of $\mathbf{G}$ provides us with the average number of glycans per protein. The end goal of glycoprofiling, however, is to elucidate the identity of particular glycoforms (P) and the concentration of each glycoform $\left(\mathbf{C}_{\mathbf{P}}\right)$. If $\mathbf{C}_{\mathbf{P}}$ is measured using an alternative technique, it is possible to calculate a unique value of $\mathbf{P}$ by solving the Diophantine matrix equation using Smith normalization. 
Given precise values of $\mathbf{G}$ and $\mathbf{C}_{\mathbf{P}}$, we want to be able to solve the following equation:

$$
\mathbf{G}=\mathbf{P}^{\mathrm{T}} \mathbf{C}_{\mathrm{P}}
$$

The matrix $\mathbf{P}$ is $i \mathbf{x} j$, where $i$ is the number of glycoforms and $j$ is the total number of glycans. $\mathbf{C}_{\mathbf{P}}$ is a $i$ component vector pertaining to the concentration of each glycoform and $\mathbf{G}$ is a $j$-component vector containing the concentration of each glycan. Assuming that both $\mathbf{G}$ and $\mathbf{C}_{\mathbf{P}}$ are known, we want to solve for the matrix $\mathbf{P}$. In order to solve the equation using the form $\mathbf{A X}=\mathbf{B}$, where $\mathbf{X}$ is the unknown, we need to rearrange the equation above by taking the transpose of both sides.

$$
\begin{gathered}
\left(\mathbf{P}^{\mathrm{T}} \mathbf{C}_{\mathrm{P}}\right)^{\mathrm{T}}=\mathbf{G}^{\mathrm{T}} \\
\mathbf{C}_{\mathrm{P}}^{\mathrm{T}} \mathbf{P}=\mathbf{G}^{\mathrm{T}}
\end{gathered}
$$

The ensuing algorithm for solving the above equation has been adapted from Greenwell and Kertzner. ${ }^{1}$

Solution algorithm:

1) Create the following matrix, where * is left blank.

$$
\left|\begin{array}{cc}
\mathbf{C}_{\mathbf{P}}^{\mathbf{T}} & \mathbf{G}^{\mathbf{T}} \\
\mathbf{I}_{i} & *
\end{array}\right|=\left|\begin{array}{cc}
\mathbf{A} & \mathbf{B} \\
\mathbf{I}_{i} & *
\end{array}\right|
$$

$i$ is equal to the number of entries in $\mathbf{C}_{\mathbf{P}}$, and $\mathbf{I}_{i}$ is the identity matrix of size $i . \mathbf{C}_{\mathbf{P}}{ }^{\mathbf{T}}$ is a (1 $\left.\mathrm{x} i\right)$ vector and $\mathbf{G}^{\mathbf{T}}$ is a $(1 \times p)$ vector.

2) Identify the smallest nonzero absolute value in $\mathbf{C}_{\mathbf{P}}^{\mathbf{T}}$. The column containing this value is referred to by $\mathrm{C}^{*}$. Perform column operations by dividing the rest of the columns by $\mathrm{C}^{*}$ and replacing the entries in each column with the remainders after division. Division between two columns is component-by-component. Continue this step until all of the entries in the first row of A are 0, with the exception of one entry (in column $\mathrm{C}^{*}$ ).

3) Divide row 1 of the augmented matrix by the only nonzero value in $\mathbf{A}$ (in column $\mathrm{C}^{*}$ ). This is will result in a value of 1 in column $\mathrm{C}^{*}$.

4) Switch the columns of $\mathbf{A}$ and $\mathbf{I}_{i}$ in the augmented matrix order to move the value of 1 into the top left (pivot) position (i.e., switch column $\mathrm{C}^{*}$ with $\mathrm{C}_{1}$ ).

The augmented matrix at the end will be of the following form:

$$
\left|\begin{array}{cc}
I_{1} O & \mathbf{V} \\
\mathbf{Q} & *
\end{array}\right|
$$

The resulting matrix $\mathbf{Q}$ can be decomposed into a vector $\mathbf{Q}_{1}$ formed from the first column of $\mathbf{Q}$ and a matrix $\mathbf{Q}_{\mathbf{2}}$ formed from the remaining columns of $\mathbf{Q}$. Moreover, in this case, $\mathbf{V}$ will be a single-row vector. The family of solutions will be described by: 


$$
\mathbf{P}=\mathbf{Q}_{1} \mathbf{V}+\mathbf{Q}_{2} \mathbf{Z}
$$

$\mathbf{Z}$ is a matrix of parameters that can vary. The entries of $\mathbf{Z}$ will be constrained given that $\mathbf{P}$ must contain nonnegative integer values. Two examples of this algorithm are provided below.

\section{Example 1}

Let

$$
\begin{aligned}
& \mathbf{P}=\left|\begin{array}{lllll}
1 & 0 & 0 & 0 & 0 \\
0 & 1 & 0 & 0 & 1 \\
1 & 0 & 0 & 1 & 0 \\
0 & 0 & 2 & 0 & 0
\end{array}\right| \\
& \mathbf{P}^{\mathrm{T}}=\left|\begin{array}{llll}
1 & 0 & 1 & 0 \\
0 & 1 & 0 & 0 \\
0 & 0 & 0 & 2 \\
0 & 0 & 1 & 0 \\
0 & 1 & 0 & 0
\end{array}\right|, \\
&
\end{aligned}
$$

Therefore $m=1, i=4$ and $p=5$. We can now form the augmented matrix in step 1:

$$
\left|\begin{array}{llll}
1 & 4 & 3 & 2 \\
1 & 0 & 0 & 0 \\
0 & 1 & 0 & 0 \\
0 & 0 & 1 & 0 \\
0 & 0 & 0 & 1
\end{array}\right|
$$

Perform the following operations: $\mathrm{C}_{2}-4 \mathrm{C}_{1} \rightarrow \mathrm{C}_{2} ; \mathrm{C}_{3}-3 \mathrm{C}_{1} \rightarrow \mathrm{C}_{3} ; \mathrm{C}_{4}-2 \mathrm{C}_{1} \rightarrow \mathrm{C}_{4}$

$$
\left|\begin{array}{cccc|ccccc}
1 & 0 & 0 & 0 & 4 & 4 & 4 & 3 & 4 \\
1 & -4 & -3 & -2 & & & & \\
0 & 1 & 0 & 0 & & & & \\
0 & 0 & 1 & 0 & & & & \\
0 & 0 & 0 & 1
\end{array}\right|
$$

Steps 3 and 4 are not needed since row 1 already contains the number 1 in the pivot position. Based on the resulting augmented matrix,

$$
\mathbf{V}=\left|\begin{array}{lllll}
4 & 4 & 4 & 3 & 4
\end{array}\right|
$$

and 


$$
\mathbf{Q}=\left|\begin{array}{cccc}
1 & -4 & -3 & -2 \\
0 & 1 & 0 & 0 \\
0 & 0 & 1 & 0 \\
0 & 0 & 0 & 1
\end{array}\right| .
$$

$\mathbf{Q}$ can be decomposed into $\mathrm{Q}_{1}$ and $\mathrm{Q}_{2}$.

$$
\mathbf{Q}_{1}=\left|\begin{array}{l}
1 \\
0 \\
0 \\
0
\end{array}\right| \text { and } \mathbf{Q}_{2}=\left|\begin{array}{ccc}
-4 & -3 & -2 \\
1 & 0 & 0 \\
0 & 1 & 0 \\
0 & 0 & 1
\end{array}\right|
$$

The solution can now be written as:

$$
\begin{gathered}
\mathbf{P}=\mathbf{Q}_{1} \mathbf{V}+\mathbf{Q}_{2} \mathbf{Z} \\
\mathbf{P}=\left|\begin{array}{l}
1 \\
0 \\
0 \\
0
\end{array}\right| \cdot\left|\begin{array}{lllll}
4 & 4 & 4 & 3 & 4
\end{array}\right|+\left|\begin{array}{ccc}
-4 & -3 & -2 \\
1 & 0 & 0 \\
0 & 1 & 0 \\
0 & 0 & 1
\end{array}\right| \cdot\left|\begin{array}{ccccc}
z_{1} & z_{2} & z_{3} & z_{4} & z_{5} \\
z_{6} & z_{7} & z_{8} & z_{9} & z_{10} \\
z_{11} & z_{12} & z_{13} & z_{14} & z_{15}
\end{array}\right| \\
\mathbf{P}=\left|\begin{array}{lllll}
4 & 4 & 4 & 3 & 4 \\
0 & 0 & 0 & 0 & 0 \\
0 & 0 & 0 & 0 & 0 \\
0 & 0 & 0 & 0 & 0
\end{array}\right|+\left|\begin{array}{ccc}
-4 & -3 & -2 \\
1 & 0 & 0 \\
0 & 1 & 0 \\
0 & 0 & 1
\end{array}\right| \cdot\left|\begin{array}{lllll}
z_{1} & z_{2} & z_{3} & z_{4} & z_{5} \\
z_{6} & z_{7} & z_{8} & z_{9} & z_{10} \\
z_{11} & z_{12} & z_{13} & z_{14} & z_{15}
\end{array}\right|
\end{gathered}
$$

The matrix below contains all possible solutions for the matrix $\mathbf{P}$. We must now restrict the solution space to all nonnegative integers.

$$
\mathbf{P}=\left|\begin{array}{ccccc}
4-4 z_{1}-3 z_{6}-2 z_{11} & 4-4 z_{2}-3 z_{7}-2 z_{12} & 4-4 z_{3}-3 z_{8}-2 z_{13} & 3-4 z_{4}-3 z_{9}-2 z_{14} & 4-4 z_{5}-3 z_{10}-2 z_{15} \\
z_{1} & z_{2} & z_{3} & z_{4} & z_{5} \\
z_{6} & z_{7} & z_{8} & z_{9} & z_{10} \\
z_{11} & z_{12} & z_{13} & z_{14} & z_{5}
\end{array}\right|
$$

We can move column-by-column to determine solutions that satisfy our conditions.

Column 1: $\left(\mathrm{z}_{1}, \mathrm{z}_{6}, \mathrm{z}_{11}\right)=(1,0,0),(0,1,0),(0,0,1),(0,0,2)$

Column 2: $\left(\mathrm{z}_{2}, \mathrm{z}_{7}, \mathrm{z}_{12}\right)=(1,0,0),(0,1,0),(0,0,1),(0,0,2)$

Column 3: $\left(\mathrm{z}_{3}, \mathrm{z}_{8}, \mathrm{z}_{13}\right)=(1,0,0),(0,1,0),(0,0,1),(0,0,2)$

Column 4: $\left(\mathrm{z}_{4}, \mathrm{z}_{9}, \mathrm{z}_{14}\right)=(0,1,0),(0,0,1)$

Column 5: $\left(\mathrm{z}_{5}, \mathrm{z}_{10}, \mathrm{z}_{15}\right)=(1,0,0),(0,1,0),(0,0,1),(0,0,2)$

Full solutions can entail any possible combinations of the above solutions, resulting in a total of $4 \times 4 \times 4 \times 4 \times 2=512$ solutions. This solution space can be further reduced by restricting the sum in each row 
of $\mathbf{P}$. Specifically, the sum of each row of $\mathbf{P}$ must be less than or equal to the total number of glycosylation sites on the protein. In this example, we will assume the number of glycosylation sites is equal to 2 .

For this particular problem, enforcing this rule reduced the number of possible solutions from 512 to 12 .

We can check two example solutions from the remaining group of 12 :

$$
\begin{aligned}
& \mathbf{P}_{1}=\left|\begin{array}{lllll}
0 & 0 & 1 & 0 & 0 \\
1 & 1 & 0 & 0 & 0 \\
0 & 0 & 1 & 1 & 0 \\
0 & 0 & 0 & 0 & 2
\end{array}\right| \rightarrow \mathbf{P}_{1}^{\mathrm{T}}=\left|\begin{array}{llll}
0 & 1 & 0 & 0 \\
0 & 1 & 0 & 0 \\
1 & 0 & 1 & 0 \\
0 & 0 & 1 & 0 \\
0 & 0 & 0 & 2
\end{array}\right| \rightarrow\left|\begin{array}{llll}
0 & 1 & 0 & 0 \\
0 & 1 & 0 & 0 \\
1 & 0 & 1 & 0 \\
0 & 0 & 1 & 0 \\
0 & 0 & 0 & 2
\end{array}\right| \cdot\left|\begin{array}{c}
1 \\
4 \\
3 \\
2
\end{array}\right|=\left|\begin{array}{c}
4 \\
4 \\
4 \\
3 \\
4
\end{array}\right|=\mathbf{G} \\
& \mathbf{P}_{2}=\left|\begin{array}{lllll}
0 & 0 & 0 & 0 & 1 \\
1 & 1 & 0 & 0 & 0 \\
0 & 0 & 0 & 1 & 1 \\
0 & 0 & 2 & 0 & 0
\end{array}\right| \rightarrow \mathbf{P}_{1}^{\mathrm{T}}=\left|\begin{array}{llll}
0 & 1 & 0 & 0 \\
0 & 1 & 0 & 0 \\
0 & 0 & 0 & 2 \\
0 & 0 & 1 & 0 \\
1 & 0 & 1 & 0
\end{array}\right| \rightarrow\left|\begin{array}{llll}
0 & 1 & 0 & 0 \\
0 & 1 & 0 & 0 \\
0 & 0 & 0 & 2 \\
0 & 0 & 1 & 0 \\
1 & 0 & 1 & 0
\end{array}\right| \cdot\left|\begin{array}{c}
1 \\
4 \\
3 \\
2
\end{array}\right|=\left|\begin{array}{c}
4 \\
4 \\
4 \\
3 \\
4
\end{array}\right|=\mathbf{G}
\end{aligned}
$$

In this case, it is not possible to solve for a single, unique value of $\mathbf{P}$ given the values of $\mathbf{G}$ and $\mathbf{C}_{\mathbf{P}}$.

\section{Example 2}

Let

$$
\begin{aligned}
& \mathbf{P}=\left|\begin{array}{llll}
0 & 0 & 0 & 1 \\
0 & 1 & 0 & 1 \\
0 & 0 & 2 & 0 \\
1 & 0 & 1 & 0 \\
1 & 1 & 0 & 0
\end{array}\right| \\
& \mathbf{P}^{\mathrm{T}}=\left|\begin{array}{lllll}
0 & 0 & 0 & 1 & 1 \\
0 & 1 & 0 & 0 & 1 \\
0 & 0 & 2 & 1 & 0 \\
1 & 1 & 0 & 0 & 0
\end{array}\right|, \mathbf{C}_{\mathbf{P}}=\left|\begin{array}{c}
1.2 \\
4.7 \\
0.3 \\
2.8 \\
8.4
\end{array}\right|, \quad \mathbf{G}=\left|\begin{array}{c}
11.2 \\
13.1 \\
3.4 \\
5.9
\end{array}\right|
\end{aligned}
$$

Therefore $m=1, i=5$ and $p=4$. We can now form the augmented matrix in step 1: 


$$
\left|\begin{array}{ccccc|cccc}
1.2 & 4.7 & 0.3 & 2.8 & 8.4 \\
1 & 0 & 0 & 0 & 0 \\
0 & 1 & 0 & 0 & 0 & 11.2 & 13.1 & 3.4 & 5.9 \\
0 & 0 & 1 & 0 & 0 \\
0 & 0 & 0 & 1 & 0 \\
0 & 0 & 0 & 0 & 1
\end{array}\right|
$$

Step 2: Column $C^{*}$ is column 3. Perform the following column operations: $C_{1}-4 C_{3} \rightarrow C_{1} ; C_{2}-$ $15 \mathrm{C}_{3} \rightarrow \mathrm{C}_{2} ; \mathrm{C}_{4}-9 \mathrm{C}_{3} \rightarrow \mathrm{C}_{4} ; \mathrm{C}_{5}-28 \mathrm{C}_{3} \rightarrow \mathrm{C}_{5}$

$$
\left|\begin{array}{ccccc|cccc}
0 & 0.2 & 0.3 & 0.1 & 0 & 11.2 & 13.1 & 3.4 & 5.9 \\
1 & 0 & 0 & 0 & 0 & & & & \\
0 & 1 & 0 & 0 & 0 & & & & \\
-4 & -15 & 1 & -9 & -28 & & & & \\
0 & 0 & 0 & 1 & 0 & & & & \\
0 & 0 & 0 & 0 & 1
\end{array}\right|
$$

We perform another set of column operations to obtain only one nonzero entry in the first row of the left side of the augmented matrix: $\mathrm{C}_{2}-2 \mathrm{C}_{4} \rightarrow \mathrm{C}_{2} ; \mathrm{C}_{3}-3 \mathrm{C}_{4} \rightarrow \mathrm{C}_{3}$

$$
\left|\begin{array}{ccccc|cccc}
0 & 0 & 0 & 0.1 & 0 & 11.2 & 13.1 & 3.4 & 5.9 \\
1 & 0 & 0 & 0 & 0 & & & & \\
0 & 1 & 0 & 0 & 0 & & & & \\
-4 & 3 & 28 & -9 & -28 & & & & \\
0 & -2 & -3 & 1 & 0 \\
0 & 0 & 0 & 0 & 1 & & & &
\end{array}\right|
$$

Step 3: Divide the first row of the augmented matrix by 0.1 in order to obtain a 1 as the only nonzero element on the left side.

$$
\left|\begin{array}{ccccc|cccc}
0 & 0 & 0 & 1 & 0 & 112 & 131 & 34 & 59 \\
1 & 0 & 0 & 0 & 0 & & & & \\
0 & 1 & 0 & 0 & 0 & & & & \\
-4 & 3 & 28 & -9 & -28 & & & & \\
0 & -2 & -3 & 1 & 0 & & & & \\
0 & 0 & 0 & 0 & 1 & & & &
\end{array}\right|
$$

Step 4: Interchange columns 1 and 4 in the augmented matrix to obtain a 1 in the pivot position. 


$$
\left|\begin{array}{ccccc|cccc}
1 & 0 & 0 & 0 & 0 & 112 & 131 & 34 & 59 \\
0 & 0 & 0 & 1 & 0 & & & & \\
0 & 1 & 0 & 0 & 0 & & & & \\
-9 & 3 & 28 & -4 & -28 & & & & \\
1 & -2 & -3 & 0 & 0 & & & & \\
0 & 0 & 0 & 0 & 1
\end{array}\right|
$$

Based on the resulting augmented matrix,

$$
\mathbf{V}=\left|\begin{array}{llll}
112 & 131 & 34 & 59
\end{array}\right|
$$

and

$$
\mathbf{Q}=\left|\begin{array}{ccccc}
0 & 0 & 0 & 1 & 0 \\
0 & 1 & 0 & 0 & 0 \\
-9 & 3 & 28 & -4 & -28 \\
1 & -2 & -3 & 0 & 0 \\
0 & 0 & 0 & 0 & 1
\end{array}\right|
$$

$\mathbf{Q}$ can be decomposed into $\mathrm{Q}_{1}$ and $\mathrm{Q}_{2}$.

$$
\mathbf{Q}_{1}=\left|\begin{array}{c}
0 \\
0 \\
-9 \\
1 \\
0
\end{array}\right| \text { and } \mathbf{Q}_{2}=\left|\begin{array}{cccc}
0 & 0 & 1 & 0 \\
1 & 0 & 0 & 0 \\
3 & 28 & -4 & -28 \\
-2 & -3 & 0 & 0 \\
0 & 0 & 0 & 1
\end{array}\right|
$$

The solution can now be written as:

$$
\begin{aligned}
& \mathbf{P}=\mathbf{Q}_{1} \mathbf{V}+\mathbf{Q}_{2} \mathbf{Z} \\
& \mathbf{P}=\left|\begin{array}{c}
0 \\
0 \\
-9 \\
1 \\
0
\end{array}\right| \cdot\left|\begin{array}{llll}
112 & 131 & 34 & 59
\end{array}\right|+\left|\begin{array}{cccc}
0 & 0 & 1 & 0 \\
1 & 0 & 0 & 0 \\
3 & 28 & -4 & -28 \\
-2 & -3 & 0 & 0 \\
0 & 0 & 0 & 1
\end{array}\right| \cdot\left|\begin{array}{cccc}
z_{1} & z_{2} & z_{3} & z_{4} \\
z_{5} & z_{6} & z_{7} & z_{8} \\
z_{9} & z_{10} & z_{11} & z_{12} \\
z_{13} & z_{14} & z_{15} & z_{16}
\end{array}\right| \\
& \mathbf{P}=\left|\begin{array}{cccc}
0 & 0 & 0 & 0 \\
0 & 0 & 0 & 0 \\
-1008 & -1179 & -306 & -531 \\
112 & 131 & 34 & 59 \\
0 & 0 & 0 & 0
\end{array}\right|+\left|\begin{array}{cccc}
0 & 0 & 1 & 0 \\
1 & 0 & 0 & 0 \\
3 & 28 & -4 & -28 \\
-2 & -3 & 0 & 0 \\
0 & 0 & 0 & 1
\end{array}\right| \cdot\left|\begin{array}{cccc}
z_{1} & z_{2} & z_{3} & z_{4} \\
z_{5} & z_{6} & z_{7} & z_{8} \\
z_{9} & z_{10} & z_{11} & z_{12} \\
z_{13} & z_{14} & z_{15} & z_{16}
\end{array}\right|
\end{aligned}
$$


The matrix below contains all possible solutions for the matrix $\mathbf{P}$. We must now restrict the solution space to all nonnegative integers.

$$
\mathbf{P}=\left|\begin{array}{cccc}
z_{9} & z_{10} & z_{11} & z_{12} \\
z_{1} & z_{2} & z_{3} & z_{4} \\
-1008+3 z_{1}+28 z_{5}-4 z_{9}-28 z_{13} & -1179+3 z_{2}+28 z_{6}-4 z_{10}-28 z_{14} & -306+3 z_{3}+28 z_{7}-4 z_{11}-28 z_{15} & -531+3 z_{4}+28 z_{8}-4 z_{12}-28 z_{16} \\
112-2 z_{1}-3 z_{5} & 131-2 z_{2}-3 z_{6} & 34-2 z_{3}-3 z_{7} & 59-2 z_{4}-3 z_{8} \\
z_{13} & z_{14} & z_{15} & z_{16}
\end{array}\right|
$$

We can move column-by-column to determine solutions that satisfy our conditions

Column 1: $\left(\mathrm{z}_{1}, \mathrm{z}_{5}, \mathrm{z}_{9}, \mathrm{z}_{13}\right)=13$ total integer solutions: $(0,36,0,0),(0,37,0,0),(0,37,0,1),(0,37,1,0),(0$, $37,2,0),(0,37,3,0),(0,37,4,0),(0,37,5,0),(0,37,6,0),(0,37,7,0),(1,36,0,0),(2,36,0,0),(2,36,1,0)$

Column 2: $\left(\mathrm{z}_{2}, \mathrm{z}_{6}, \mathrm{z}_{10}, \mathrm{z}_{14}\right)=18$ total integer solutions: $(0,43,0,0),(0,43,1,0),(0,43,2,0),(0,43,3,0)$, $(0,43,4,0),(0,43,5,0),(0,43,6,0),(1,42,0,0),(1,43,0,0),(1,43,0,1),(1,43,1,0),(1,43,2,0),(1,43,3,0)$, $(1,43,4,0),(1,43,5,0),(1,43,6,0),(1,43,7,0),(2,42,0,0)$

Column 3: $\left(\mathrm{z}_{3}, \mathrm{z}_{7}, \mathrm{z}_{11}, \mathrm{z}_{15}\right)=1$ integer solution: $(0,11,0,0)$

Column 4: $\left(\mathrm{z}_{4}, \mathrm{z}_{8}, \mathrm{z}_{12}, \mathrm{z}_{16}\right)=3$ integer solutions: $(0,19,0,0),(1,19,0,0),(1,19,1,0)$

Total number of possible solutions $=13 \times 18 \times 1 \times 3=\mathbf{7 0 2}$.

Given our assumption that the glycoforms in the mixture have a maximum of 2 glycosylation sites, we can eliminate the values of $\mathbf{P}$ in which the sum of a given row is greater than 2. For this particular problem, enforcing this rule reduced the number of possible solutions from 702 to 1:

$$
\mathbf{P}=\left|\begin{array}{llll}
0 & 0 & 0 & 1 \\
0 & 1 & 0 & 1 \\
0 & 0 & 2 & 0 \\
1 & 0 & 1 & 0 \\
1 & 1 & 0 & 0
\end{array}\right|
$$

In this case, knowledge of $\mathbf{G}$ and $\mathbf{C}_{\mathbf{P}}$ enabled the solution of a unique value of $\mathbf{P}$, identifying all of the glycoforms in the mixture.

\section{References}

1 Greenwell, R. N. \& Kertzner, S. Solving linear diophantine matrix equations using the smith normal form (more or less). International Journal of Pure and Applied Mathematics 55, 49-60 (2009). 